\title{
Special Issue on Automotive Lightweight
}

\author{
A. Erman Tekkaya ${ }^{1} \cdot$ Junying Min $^{2}$
}

Published online: 31 August 2020

(c) China Society of Automotive Engineers (China SAE) 2020

Lightweight is one of the core technologies to realize energy saving, to improve the safety and driving performance of vehicles, and to provide added passenger comfort as well. It is of special significance for increasing cruising range of electric or hybrid-powered vehicles. Beyond these, lightweight components use less mass and hence reduce the primary energy requirement imbedded in the component. Therefore, lightweight has always been a central topic and an important direction in the automotive field whether in the past or in the future.

Lightweight promotes the increasing application of lightweight materials, such as advanced high-strength steels, $\mathrm{Al}$ and $\mathrm{Mg}$ alloys, carbon fiber-reinforced polymers, and hybrid metal-polymer composites, which requires innovative forming and joining processes to produce and assemble automotive components. The concept of the right material in the right place breeds multi-material car body, and philosophy is more needed than in the past to balance the maximal weight reduction, service performance, and cost of vehicles and to comprehensively employ lightweight materials, lightweight design strategies as well as lightweight processes.

We hope this special issue will promote technological progress in automotive lightweight. Two articles address how to represent lightweight design by innovative forming processes and seek to address a hot issue in sheet metal forming; three articles are related to hybrid or dissimilar materials joining technologies; two articles deal with structural designs of lightweight electric vehicles; one article proposes an index to quantitatively evaluate the degree of automotive lightweight; and one article introduces and investigates lightweight materials, i.e., metal-FRP composites.

Junying Min

Junying.Min@tongji.edu.cn

A. Erman Tekkaya

Erman.Tekkaya@iul.tu-dortmund.de

1 Institute of Forming Technology and Lightweight, Components, TU Dortmund University, 44227 Dortmund, Germany

2 School of Mechanical Engineering, Tongji University, Shanghai 201804, China
The successful publication of this special issue is inseparable from the support of many parties in this difficult time of Coronavirus pandemic. First of all, we would like to deeply thank the authors for sharing their latest and excellent research results and timely submitting their original and revised manuscripts. We are indebted to the involved reviewers for their time and suggestive review comments that definitely increased the overall quality of the papers. China Automotive Lightweight Technology Innovation Strategic Alliance (CALA) is gratefully acknowledged due to the support in collecting manuscripts and in the organization of the international symposium dedicated to this special issue. Finally, we are grateful to the colleagues from the editorial office for providing numerous help and suggestions without reservations during the organization and production of this special issue.

\section{Highlights of Articles in the Special Issue}

The paper "Lightweight in Automotive Components by Forming Technology" by Stephan Rosenthal, Fabian Maaß, Mike Kamaliev, Marlon Hahn, Soeren Gies, and A. Erman Tekkaya [Paper No. 1] gives a comprehensive review on the forming processes producing light automotive components/ structures and discusses innovative processes in terms of the following classification of lightweight design: material, structure, and system lightweight design.

Yong Sun, Vladimir Luzin, Yixin Duan, Rameshkumar Varma, Lei Shi, and Matthias Weiss, by composing "Forming-Induced Residual Stress and Material Properties of RollFormed High-Strength Steels" [Paper No. 2], present an experimental and numerical study on residual stresses of roll-formed high-strength martensitic steel, and the variation of mechanical properties of a roll-formed automotive part is discussed.

Mingfeng Li, Yanjun Wang, Zhen Niu, and Shanglu Yang, the authors of "Study on the Weld-Bonding Process Optimization and Mechanical Performance of Aluminum Alloy Joints" [Paper No. 3], optimize the hybrid weldbonding process to achieve superior weld nugget quality and more stable mechanical properties of $\mathrm{Al}$ alloy joints. 
In "Effects of Standoff Distance on Magnetic Pulse Welded Joints Between Aluminum and Steel Elements in Automobile Body" [Paper No. 4], Junjia Cui, Shaoluo Wang, Wei Yuan, and Guangyao Li present a novel joining process of dissimilar sheet metals through magnetic pulse welding, and the correlation between interfacial microstructure and mechanical performance of joints is revealed.

Yunwu Ma, Sizhe Niu, He Shan, Yongbing Li, and Ninshu Ma, in writing "Impact of Stack Orientation on SelfPiercing Riveted and Friction Self-Piercing Riveted Aluminum Alloy and Magnesium Alloy Joints" [Paper No. 5], compare two riveting processes in detail when joining dissimilar light alloys. It is found there is a preference in stack orientation of the two dissimilar sheet metals.

Dengfeng Wang, Chong Xie, Yuchang Liu, Wenchao $\mathrm{Xu}$, and Qi Chen composed "Multi-objective Collaborative Optimization for the Lightweight Design of an Electric Bus Body Frame" [Paper No. 6], achieving lightweight effect of an electric bus through multi-objective collaborative lightweight design, where structural strength, stiffness, modal, and rollover safety of the electric bus are comprehensively considered.

Zitong Shi, Guanhua Chen, Lingxiao Zhu, Jie Li, and Yong Xia, authors of "Sandwich Structure Design of a Cooling Fin for Battery Modules Against Impact Loads" [Paper No. 7], numerically and experimentally investigate the mechanical behavior of a lithium-ion battery module under impact load and propose a design of protection structure utilizing the energy absorbing ability of cooling fins.

Jun Li, Ligang Wang, Yunxia Chen, Hongzhou Lu, and Haitao Jiang, in "Research and Application of Lightweight Index for Passenger Cars" [Paper No. 8], introduce an lightweight index to assess the lightweight level and verify it through statistical analysis on thousands of internal combustion engine vehicles and battery electric vehicles.

The last paper comes from Ye Lin, Junying Min, Hao Teng, Jianping Lin, Jiahao Hu, and Nanjie Xu. In "Flexural Performance of Steel-FRP Composites for Automotive Applications" [Paper No. 9], they comprehensively review the application of metal-FRP composites, especially in automotive industry, and investigate the mechanical performance of steel-FRP composites fabricating from steel and various fiber-reinforced polymers subject to three-point bending.

\section{Dr. A. Erman Tekkaya \\ Dr. Junying Min}

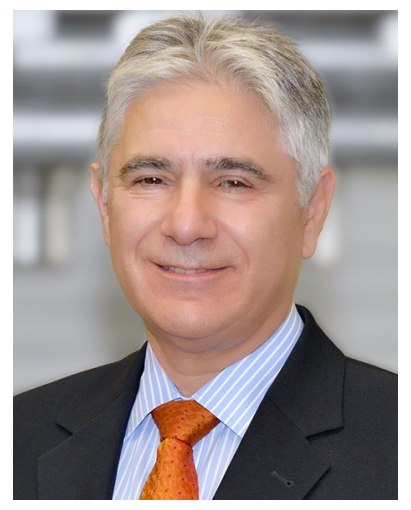

Dr. A. Erman Tekkaya is currently professor and the director of the Institute for Metal Forming and Lightweight Components (IUL) at the TU Dortmund University in Dortmund, Germany. His research interests cover fundamentals and technology of novel metal forming processes as well as material characterization for modeling the material behavior during plastic deformations. Some of the current research projects are on thermomechanical sheet forming processes, profile bending, hot extrusion, impact extrusion, damage-controlled metal forming, the plane torsion test, remote material testing, and metal forming including augmented reality applications. The International Prize for Research \& Development in Precision Forging of the Japan Society for Technology of Plasticity has been awarded to him in 2014. Dr. Tekkaya is a fellow of the International Academy for Production Engineering (CIRP) and member of the Deutsche Gesellschaft für Materialkunde (DGM). He is the Editor-in-Chief of the CIRP Annals, Editor-in-Chief of Advances in Industrial and Manufacturing Engineering (AIME). He has published over 215 peer- reviewed papers, several books and has over 30 patents granted or filed.

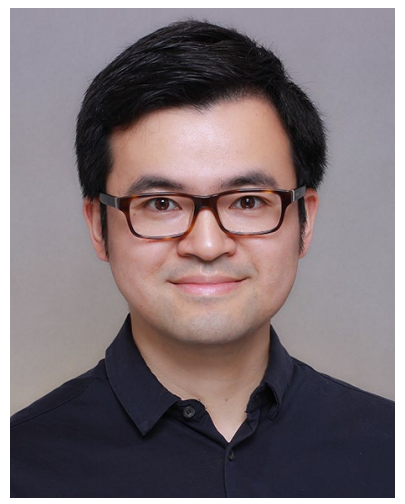

Dr. Junying Min is a professor at the School of Mechanical Engineering and the vice director of the Sino-German Center for Mechanical Engineering of Tongji University. His research interests include advanced forming processes, material characterization/modeling, dissimilar materials joining and hybrid components. He was a postdoc at the University of Hawaii and University of Michigan from 2013 to 2014. During that period, he was also a visiting scientist at the General Motors Global R\&D Center. He conducted research at the Ruhr-University Bochum from 2015 to 2017 as an Alexander von Humboldt fellow. He received Hiwin Doctoral Dissertation Award in 2014. He sits on the editorial board of Automotive Innovation (AUIN) and Advances in Industrial and Manufacturing Engineering (AIME). He has published more than 100 peer-reviewed papers, over 30 in the JCR Q1 journals with international reputation, one book with his co-authors, and he holds over 10 invention patents. 